\title{
Automated summit spot height generation for modern topographic mapping
}

\author{
S.T. Arundel ${ }^{\text {a, } *}$, A. Chan ${ }^{\text {b }}$ \\ ${ }^{a}$ U.S. Geological Survey, sarundel@usge.gov \\ ${ }^{b}$ Missouri University of Science and Technology, achgb@mst.edu \\ * Corresponding author
}

Keywords: U.S. Topo, Summits, 3DEP, Lidar, GNIS

\begin{abstract}
:
Spot elevations published on historical U.S. Geological Survey (USGS) topographic maps were established as needed to enhance information imparted by the quadrangle's contours. In addition to a number of other features like road intersections, section corners, lakes and water wells, labels were routinely placed on mountain peaks, local high points, passes and saddles. While some elevations were established through field survey triangulation, many were "dropped” during photogrammetric stereocompilation. The average accuracy, which varied with method, terrain, and map vintage, was on the order of $+/-10$ feet.

Today, inexpensive consumer devices deliver comparable accuracy using the Global Positioning System (GPS). Professional equipment can achieve even better accuracies. These methods have replaced the field- and fundingintensive traditional triangulation methods in the geographic sciences. However, since GPS measurements require visiting the feature location, a national dataset containing high-accuracy spot elevations has not yet been created. Consequently, modern US Topo maps are devoid of mountain peak or other spot elevations. Additionally, no government agency is currently mandated to create one.
\end{abstract}

Still, US Topo map users continue to demand the display of spot heights, particularly mountain peak elevations. As lidar data are collected through the 3D Elevation Program (3DEP), the source for higher accuracy spot heights increases. Therefore, a pilot study was conducted to evaluate the feasibility of automatically generating elevation values at summits named in the Board on Geographic Name’s Geographic Names Information System (GNIS), using 3DEP data. As the 3DEP incorporates more lidar data, these values should become increasingly more accurate.

The first step in the automation process involved "snapping” GNIS summits to the highest and most accurate nearby point (pixel) in the 3DEP one-third arc second seamless dataset (1/3 a-s). Development of this step was completed in a prior study and will be reported elsewhere. In Step 2, a process similar to Step 1 was implemented to identify the highest pixel in the 3DEP one-meter dataset (1-m) from an area defined by a one-pixel buffer around the snapped point pixel. After finding the highest pixel in the 1-m dataset, the same process for Step 2 was repeated on the lidar point cloud dataset (LPC). In the latter case, the highest lidar point within the buffered area was chosen where there were more than one, which was the case in most instances.

In the case that the next higher resolution data were unavailable, the summit spot elevation was set to the value obtained from the lower resolution layer. Hence, if 1-m data were unavailable, but LPC data did exist, the program in its current state was unable use the higher resolution data. This is due to the fact that the 1-m data are employed to drive the software to a more precise location of the summit. Without that intermediate step the method fails to identify the correct LPC point. Work in progress is aimed at overcoming this disadvantage, which is particularly important until the time when the 1-m dataset is populated for the entire nation. The National Geospatial Program estimates the completion of lidar acquisition by 2023. Processing from acquisition to update of the 1-m dataset, including quality assessment and revisions, currently requires between two and three years.

Resulting elevation values are compared to those published by Peakbagger.com and TOPOZONE.com. Preliminary results from 40 summits indicate that values derived from lidar are generally higher, whereas those populated from the $1 / 3$ arc-second are generally lower. A thorough understanding of these relationships will require the evaluation of more points. 